\title{
A rare case of acute megakaryoblastic leukemia with orbital chloroma in a non-Down syndrome child
}

\begin{abstract}
Acute megakaryoblastic leukemia (AMKL) is a rare disease accounting for $7 \%-10 \%$ of acute myeloid leukemia (AML) in children. It is uncommon in children without Down syndrome (DS). Orbital chloroma is usually associated with AML M2, M4, and M5. Herein, we report the case of a 22-month-old female who was diagnosed to have AMKL with orbital chloromas and without DS. Morphology and the initial panel of immunophenotyping were inconclusive and the presence of orbital chloromas added to the ambiguity. The presence of CD61 expression and marrow fibrosis supported by hyperdiploidy helped us clinch the diagnosis. Hence, comprehensive analysis of morphology, immunophenotyping, and cytogenetics is warranted to make an accurate diagnosis of AMKL.
\end{abstract}

Keywords: Acute megakaryoblastic leukemia, orbital chloroma, hyperdiploidy, immunophenotyping

\section{INTRODUCTION}

Acute megakaryoblastic leukemia (AMKL) is a rare disease accounting for $7 \%-10 \%$ of acute myeloid leukemia (AML) in children. Hence, of about 500 children in the United States and seventy in the United Kingdom diagnosed with AML each year, only 50 and 7 children respectively are likely to have AMKL. ${ }^{[1]}$ Children with Down syndrome (DS) have 400-500 times higher incidence of AMKL compared to those without it. ${ }^{[2]}$ In non-DS children, diagnosis is often difficult because of indistinct clinical and morphological features. Moreover, the commonly applied panel of antibodies for immunophenotyping may often be nondiagnostic, and there are no characteristic cytogenetic abnormalities other than $t(1 ; 22)$ which is present only in $20 \%$ of patients beyond infancy. ${ }^{[3]}$

Herein, we report a case of de novo AMKL in a non-DS child who in addition to all these diagnostic challenges also had orbital chloroma and hyperdiploid karyotype.

\section{CASE REPORT}

A 22-month-old female child presented to our institute, with complaints of fever and progressive pallor for the past

\begin{tabular}{|l|l|}
\hline \multicolumn{2}{|c|}{ Access this article online } \\
\hline \multirow{2}{*}{$\begin{array}{l}\text { Website: } \\
\text { www.asjo.in }\end{array}$} & Quick Response Code \\
\hline DOI: & $\square$ \\
10.4103/2454-6798.209322 & \\
\hline
\end{tabular}

25 days associated with decreased activity and progressively increasing swelling of the left upper lid for 10 days. On examination, the child had pallor, hepatosplenomegaly, with the presence of diffuse soft and nontender swelling of the left upper eyelid [Figure 1] and no significant lymphadenopathy. Investigations revealed hemoglobin of $6 \mathrm{~g} / \mathrm{dl}$; total leukocyte count of $74.4 \times 10^{9} / \mathrm{L}$ with $80 \%$ of blasts, polymorphs $11 \%$, and lymphocytes $7 \%$ and platelets of $65.0 \times 10^{9} / \mathrm{L}$. Bone marrow aspirate was hypercellular with $69 \%$ blasts that were small in size with scant agranular cytoplasm which was negative for myeloperoxidase. Hence, an initial provisional diagnosis of ALL was considered. However, blasts were negative for CD19, 20, 79a, 10, 73 (B cell); cyCD3, CD2, 5, 7, 1a, 4, 8 (T cell), CD117, 13 (myeloid); CD11c, 64 (monocytic), CD56, 16 (NK cell), CD41a (megakaryocytic), and other (CD34, 38, 123, 14, HLA-DR, TdT) markers while being positive for

\section{Sahitya Koneru, Sandeep Jain, Gauri Kapoor Department of Pediatric Hematology and Oncology, Rajiv Gandhi Cancer Institute and Research Centre, New Delhi, India \\ Address for correspondence: Dr. Gauri Kapoor, Department of Pediatric Hematology and Oncology, Rajiv Gandhi Cancer Institute and Research Centre, New Delhi - 110 085, India. E-mail: kapoor.gauri@rgcirc.org}

This is an open access article distributed under the terms of the Creative Commons Attribution-NonCommercial-ShareAlike 3.0 License, which allows others to remix, tweak, and build upon the work non-commercially, as long as the author is credited and the new creations are licensed under the identical terms.

For reprints contact: reprints@medknow.com

How to cite this article: Koneru S, Jain S, Kapoor G. A rare case of acute megakaryoblastisc leukemia with orbital chloroma in a non-Down syndrome child. Asian J Oncol 2017;3:81-3. 


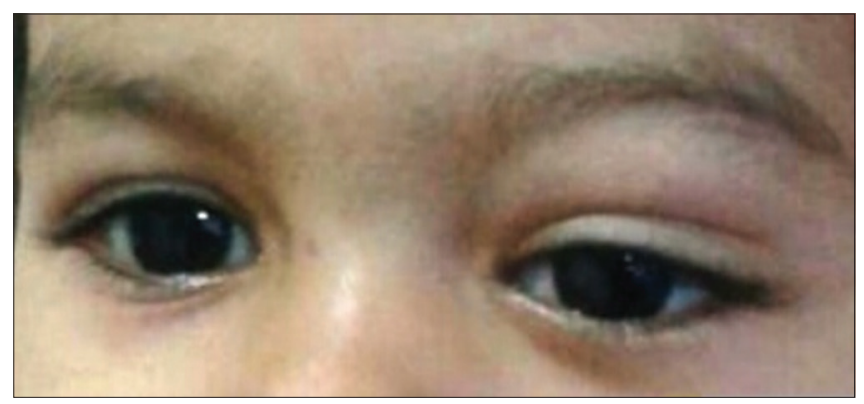

Figure 1: Ptosis of left eye due to orbital chloroma. An ill-defined, soft and nontender swelling of left upper eyelid is present leading to ptosis

CD36 (bright), CD45 (dim), CD58 (bright), and CD61 (dim). Bone marrow biopsy showed increased marrow reticulin and marked proliferation of megakaryocytes with few dysplastic forms along with immature cells. Karyotype was hyperdiploidy (57XX) with tetrasomy 2 , trisomy 6,8 , 10,19 , and 20 ; trisomy $21, \mathrm{t}(1 ; 22)$ and $\mathrm{t}(4 ; 11)$ were not observed. Computed tomography scan of the head revealed two lobulated soft tissue density lesions in the left orbit suggestive of chloromas [Figure 2a and b].

\section{DISCUSSION}

AMKL is often not suspected in non-DS children due to its rarity and difficult diagnosis. A presumptive diagnosis of AML M7 is considered if there is morphological evidence of clustering of blasts, micromegakaryocytes or dysplastic immature megakaryocytes, cytoplasmic blebbing, and platelet budding in bone marrow. However, these features are uncommon in pediatric patients, as was in the present case. Common differential diagnoses of AMKL include ALL, AML M0 and M6 which are often not distinguishable by morphological and cytochemical studies. The choice of appropriate immunologic markers such as CD 41a, CD61, $\mathrm{CD} 42 \mathrm{~b}$, and glycophorin $\mathrm{A}$ in addition to conventional myeloid, monocytic, and lymphoid markers are crucial for confirmation of diagnosis. In our case, the absence of all $\mathrm{B}$ and $\mathrm{T}$ markers virtually eliminated the possibility of lymphoid malignancy. The absence of myeloid and monocytic markers narrowed our diagnosis to AML M0, M6, or M7. The absence of CD41 and glycophorin A expression made diagnosis more difficult. The presence of marrow fibrosis and CD61 expression on repeat biopsy specimen helped us clinch the diagnosis. Platelet aggregates on the surface of megakaryoblasts may give a false impression of CD41 and CD61 positivity. Hence, cytoplasmic expression of these antigens is more reliable. Therefore, cytoplasmic expression of CD61 by permeabilization of the cell membrane and flow cytometry are recommended in all cases of AML with undifferentiated morphology and negative cytochemistries to differentiate AML M7 from AML M0. Comparison of

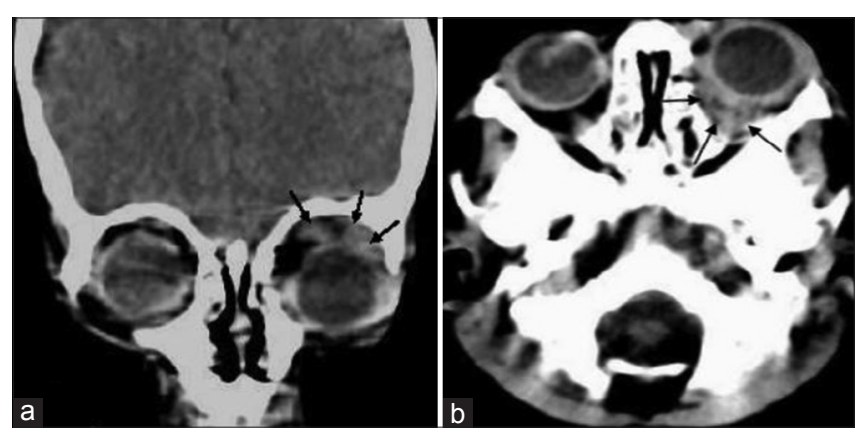

Figure 2: Computed tomography head showing two lobulated soft tissue density lesions in left orbit. (a) Chloroma, $2 \mathrm{~cm} \times 0.8 \mathrm{~cm}$, involving left lacrimal gland in superior peri-orbital space of with extension into upper eyelid. (b) Second lesion, $2.4 \mathrm{~cm} \times 0.9 \mathrm{~cm}$, in retro-orbital space of same side

these three markers have shown that CD42 is the least sensitive (absent in early megakaryoblast), CD41a is the most sensitive but least specific while CD61 is the most specific marker of megakaryocytic differentiation. ${ }^{[4]}$ Cross-linkage expression of these lineage-restricted antigens may be observed in AML M6 and M7 due to a common progenitor, which may lead to ambiguity in diagnosis..$^{[5]}$

The presence of orbital chloroma acted as a red herring as it is more commonly observed with M2, M4, and M5 FAB subtypes of AML. Although extramedullary disease in AMKL is a known entity, orbital chloroma is rare. It was reported in $<7 \%$ of pediatric AMKL cases in a large retrospective international series. ${ }^{[6]}$ Interestingly, over-expression of Apollon, an anti-apoptotic gene located on chromosome 2 has been associated with the occurrence of extramedullary disease. ${ }^{[7]}$ Although the leukemia karyotype in the present case had tetrasomy of chromosome 2, Apollon protein expression was not tested for. The other karyotype abnormalities observed in the present case, including hyperdiploidy, though reported in AMKL have not been reported in association with orbital chloroma or extramedullary disease.

\section{CONCLUSION}

Diagnosis of AMKL is often difficult due to its rarity and biological heterogeneity. Accurate Identification of this subtype is important from therapeutic aspect also, AMKL is considered as a high-risk disease in many protocols and is an indication for allogeneic bone marrow transplantation in first remission. Therefore, a high index of suspicion and careful interpretation of morphology, immunophenotyping, and cytogenetics is warranted to make an accurate and early diagnosis in the absence of characteristic features.

\section{Financial support and sponsorship}

Nil. 


\section{Conflicts of interest}

There are no conflicts of interest.

\section{REFERENCES}

1. Athale UH, Razzouk BI, Raimondi SC, Tong X, Behm FG, Head DR, et al. Biology and outcome of childhood acute megakaryoblastic leukemia: A single institution's experience. Blood 2001;97:3727-32.

2. Hama A, Yagasaki H, Takahashi Y, Nishio N, Muramatsu H, Yoshida N, et al. Acute megakaryoblastic leukaemia (AMKL) in children: A comparison of AMKL with and without Down syndrome. Br J Haematol 2008;140:552-61.

3. Bernstein J, Dastugue N, Haas OA, Harbott J, Heerema NA, Huret JL, et al. Nineteen cases of the $\mathrm{t}(1 ; 22)(\mathrm{p} 13 ; \mathrm{q} 13)$ acute megakaryblastic leukaemia of infants/children and a review of 39 cases: Report from a $\mathrm{t}(1 ; 22)$ study group. Leukemia 2000;14:216-8.

4. Käfer G, Willer A, Ludwig W, Krämer A, Hehlmann R, Hastka J. Intracellular expression of CD61 precedes surface expression. Ann Hematol 1999;78:472-4.

5. Behm FG, Campana D. Immunophenotyping. In: Pui CH, editor. Childhood Leukemias. $1^{\text {st }}$ ed. Cambridge: Cambridge University Press; 1999. p. 111-44.

6. Inaba $\mathrm{H}$, Zhou Y, Abla O, Adachi S, Auvrignon A, Beverloo HB, et al. Heterogeneous cytogenetic subgroups and outcomes in childhood acute megakaryoblastic leukemia: A retrospective international study. Blood 2015;126:1575-84.

7. Sung KW, Choi J, Hwang YK, Lee SJ, Kim HJ, Lee SH, et al, Overexpression of Apollon, an antiapoptotic protein, is associated with poor prognosis in childhood de novo acute myeloid leukemia. Clin Cancer Res 2007;13:5109-14. 